\title{
Screening Wild Oat Accessions from Morocco for Resistance to Puccinia coronata
}

M. Y. A. Tan and M. L. Carson, United States Department of Agriculture-Agricultural Research Service, Cereal Disease Laboratory, St. Paul, MN 55113

\begin{abstract}
Tan, M. Y. A., and Carson, M. L. 2013. Screening wild oat accessions from Morocco for resistance to Puccinia coronata. Plant Dis. 97:1544-1548.

Here, we report the screening of 332 new accessions of 11 different wild oat (Avena) species from the United States Department of Agriculture National Small Grains Collection in Aberdeen, ID, for resistance to crown rust disease, caused by Puccinia coronata f. sp. avenae. This collection originated from Morocco and includes Avena agadiriana, A. atlantica, A. barbata, A. damascena, A. eriantha, A. hirtula, A. longiglumis, A. magna, A. murphyi, A. sterilis, and A. wiestii. After screening this collection with a highly diverse population of $P$. coronata f. sp. avenae, 164 accessions (49\%) were rated moderately resistant to resistant in the preliminary screen at the seedling stage and 181 accessions (55\%) rated moderately resistant to highly

resistant in the adult plant stage. Although none of the accessions showed a highly resistant response consistently in the seedling stage, 20 accessions did display a highly resistant response in the adult plant stage. Both seedling as well as adult plant resistance was found in 150 (45\%) of the accessions. Virulence in P. coronata has been reported to all resistance genes currently being used in North American oat cultivars. The new resistance sources presented here are potentially new sources for future breeding. The resistance found in 52 accessions of $A$. magna and A. murphyi with the AACC genome is especially valuable because these should be relatively easy to transfer into hexaploid $A$. sativa.
\end{abstract}

The most damaging threat to domesticated oat (Avena sativa $\mathrm{L}$.) production comes from crown rust, caused by the fungus Puccinia coronata f. sp. avenae W.P. Fraser \& Ledingham. All countries that grow oat are affected by crown rust (21). Breeding cereals for resistance against rust has a long history, beginning as early as 1887 (14). Compared with other plant-pathogen systems, an abundance of over 100 crown rust resistance $(P c)$ genes in oat have been identified to date (A. Roelfs, personal communication; http://www.ars.usda.gov/Main/docs.htm?docid=10342). The first oat crown rust resistance $(R)$ genes were identified in cultivated oat (A. sativa) in the early 20th century. Resistance is usually expressed in the seedling stage and considered to be race specific and on a gene-for-gene basis. The population structure of $P$. coronata can shift rapidly due to the pathogen's ability to reproduce sexually on its alternate host, Rhamnus spp. Mutation levels are additionally extremely high outside of this sexual cycle (21). Consequently, and because cultivated oat had a narrow genetic base in North America, all deployed $P c$ genes from A. sativa proved to be ineffective by the 1950s (14). Breeders then turned to exotic sources of resistance in wild Avena spp. The hexaploid wild animated oat, A. sterilis, was first used as a source because of its crossability with cultivated oat (8). However, the initial euphoria caused by an apparent abundance of new $P c$ genes soon turned to disappointment. After widespread deployment, the new $P c$ genes proved no more durable than the resistance of $A$. sativa had been. At the present time, virulence to all described seedling $P c$ genes has been found in North America $(3,5)$.

New sources of resistance would greatly benefit the breeding efforts for resistance to crown oat, because effective $R$ genes in the

Corresponding author: M. L. Carson, E-mail: mcarson@umn.edu

* The $e$-Xtra logo stands for "electronic extra" and indicates that a supplementary table is available online.

Accepted for publication 29 May 2013.

http://dx.doi.org/10.1094/PDIS-12-12-1150-RE

This article is in the public domain and not copyrightable. It may be freely reprinted with customary crediting of the source. The American Phytopathological Society, 2013. hexaploid species have been exhausted. Approximately 30 Avena spp. have been recognized, ranging in ploidy level between diploid and hexaploid (9). The United States Department of AgricultureAgricultural Research Service National Small Grains Collection (NSGC) currently holds 11,294 wild oat accessions covering 21 oat species. Other germplasm collections outside of the United States also have substantial Avena collections. Wild Avena spp., however, particularly the diploid and tetraploid species, remain a largely untapped sources of new $P c$ genes. The diploid black oat, A. strigosa, has been used with some success as a source of resistance to crown rust, notably in the breeding programs in Winnipeg, Manitoba, and the University of Wisconsin. The wild tetraploid A. magna was the source of Pc91 now in 'HiFi', released by North Dakota State University in 2001 (16), and subsequently followed by 'Souris' in 2005, 'Rockford' in 2008, 'Newburg' in 2011, and 'Jury' in 2012, which increased selection pressure for virulence to $P c-91$ in the crown rust population.

Recent screenings of the tetraploid wild oat species $A$. barbata for crown rust resistance resulted in the identification of 170 accessions with both seedling and adult resistance $(3,4)$. These studies indicated that $A$. barbata resistance occurred more frequently in accessions originating from western Mediterranean countries. Loskutov, who has studied crown rust resistance in 22 different oat species obtained from the Vavilov Institute of Plant Industry, concluded that resistance most frequently occurred in accessions from North African countries such as Tunisia, Algeria, and Morocco (11). The center of origin of the genus Avena is located in the Mediterranean region and there is thought to be a center of diversity in the western part of the Mediterranean region: Morocco, Spain, and Algeria (12). In this, study we report the screening for oat crown rust resistance of 332 accessions originating from Morocco, which became available in 2009 at the NSGC. The accessions cover 11 different wild oat species. Resistance to crown rust was tested at the seedling stage and confirmed at the adult plant stage. We focused on identifying accessions with resistance at both stages because this type of resistance has the most practical use in the field.

\section{Materials and Methods}

Plant materials. All 332 wild oat accessions (Supplementary Table 1), originating from Morocco, were obtained from the NSGC 
in Aberdeen, ID. Accessions were originally collected between 1985 and 1989 and became available in 2009 at the NSGC. Eleven different wild oat species are represented (Table 1), including diploids (A. atlantica, A. damascena, A. eriantha, A. hirtula, A. longiglumis, and A. wiestii), tetraploids (A. agadiriana, A. barbata, A. magna, and A. murphyi), and two hexaploid accessions (A. sterilis). For 29 accessions (Avena spp.), the species designation was undetermined.

Inoculations. All inoculations were performed with a bulk inoculum of $P$. coronata $\mathrm{f}$. sp. avenae derived from the Matt Moore buckthorn nursery (University of Minnesota, St. Paul). The buckthorn nursery supports a sexually recombining population of $P$. coronata due to the presence of its alternate host, the common buckthorn (Rhamnus cathartica L.). The inoculum collected from the buckthorn nursery is composed of a complex mix of races: virulence to almost all known $P c$ genes can be detected in any given year (3). Only broad-spectrum resistance in the accessions should be selected with the bulk inoculum in contrast to screening with a single race isolate (17). Urediniospores were collected from the buckthorn nursery at St. Paul in 2010 and in 2011 and multiplied in the greenhouse on 'Starter', which has no known $P c$ genes. After harvesting the urediniospores from Starter with a vacuum collector, the fresh urediniospores were immediately suspended in a light mineral oil (Soltrol 170; Phillips Petroleum Co.). The concentration was adjusted to approximately $1 \times 10^{6} \mathrm{spores} / \mathrm{ml}$ and was sprayed over leaves of seedling or adult plants using a spray atomizer until the leaves were lightly covered. Inoculated plants were air dried and incubated in a dark dew chamber overnight (16 h) to allow for germination of the urediniospores and infection (18). Plants were then transferred to either the greenhouse for initial screening or growth chamber for subsequent testing.

Disease testing. For the initial screening of the accessions, approximately 10 seeds from each of the 332 oat accessions were planted in $7-\mathrm{cm}^{2}$ pots filled with vermiculite and placed in the greenhouse. To ensure the effectiveness of the inoculation, 1 of every 12 pots was a susceptible control containing 'Marvelous'. Seedlings were inoculated at the first-leaf stage (10 days after planting) and incubated as described above, with fresh bulk inoculum derived from the 2010 St. Paul buckthorn nursery and then transferred back to the greenhouse. The seedlings were scored for disease reaction 12 days after inoculation using a qualitative scale of $\mathrm{S}, \mathrm{MS}, \mathrm{MR}, \mathrm{R}$, and HR, where reaction phenotypes are $\mathrm{S}=$ susceptible, large to moderately large pustules with little or no chlorosis; $\mathrm{MS}=$ moderately susceptible, moderately large pustules surrounded by extensive chlorosis; $\mathrm{MR}=$ moderately resistant, small pustule surrounded by chlorosis; $\mathrm{R}=$ resistant, chlorotic or necrotic flecking; and HR = highly resistant, no visible reaction (3). Of each accession that scored as MR to HR, a single seedling was selected for seed generation and transferred to "cone-tainers" (cones) in trays $(21 \mathrm{~cm}$ deep by $3.8 \mathrm{~cm}$ in diameter) filled with a pasteurized soil mix. With accessions with mixed phenotypes, only a seedling of the highest resistance class within that accession was selected. All selected seedlings were reinoculated again after transplanting with fresh bulk inoculum to confirm the resistance phenotype seen at the first leaf stage. The selected seedlings were then allowed to grow to maturity to generate seed for further resistance testing of offspring. Two S1 plants per each R accession were evaluated at the seedling and adult plant stage. Seed were sown in cones filled with pasteurized soil and the cones were randomized. Marvelous was used as a control to check effective inoculation, with 1 in every 14 cones occupied by Marvelous. The cones were placed in a growth cabinet with a 16-h photoperiod and a temperature of 16 to $22^{\circ} \mathrm{C}$ (night to day, respectively). The primary leaves of the seedlings were inoculated as described before but with fresh urediniospores from the 2011 St. Paul buckthorn nursery, again multiplied and collected on Starter. Inoculum from 2011 was used instead of inoculum from 2010 to select against resistance where new virulence might have emerged against in 2011. Resistance phenotypes were recorded after 12 days post inoculation. In cases where the resistance phenotype differed between the two replica- tions, only the resistance phenotype of the most susceptible is reported. Seedlings were then allowed to grow to the jointing or flag-leaf stage, at which point the adult plants were inoculated with the 2011 inoculum and incubated as described, transferred back to the growth chamber, and evaluated 12 days after inoculation. Disease symptoms on seedling and adult plant level were evaluated using the qualitative scale of S, MS, MR, R, and $\mathrm{HR}$, as described above.

\section{Results}

The 332 Moroccan wild oat accessions were first tested for seedling resistance in an initial screen with bulk inoculum of $P$. coronata f. sp. avenae from the 2010 St. Paul buckthorn nursery. Disease reactions ranged from a fully $S$ to an HR phenotype. This initial screen resulted in 224 accessions $(67 \%)$ that rated MR to HR. The 108 (33\%) accessions that tested MS to S were excluded from further resistance testing. Most accessions (75\%) showed a homogeneous phenotype over all of the approximately 10 seedlings of that accession; however, variation in resistance phenotype was observed in 83 accessions. Of all accessions with at least MR, one seedling was selected for retesting and seed generation. Although they had tested MR in the initial screen, 16 accessions were excluded from further analysis because they tested MS after reinoculation. Two more accessions were excluded because they were MS or S when they had initially tested R or HR. We assume that the initial resistance phenotypes of these particular accessions were observed due to either an inoculation error or seed mix-up. Ultimately, 208 accessions were used for seed generation and subsequent phenotyping.

Two S1 offspring of each accession selected in the initial screen were tested for both seedling and adult plant resistance in the controlled environment of a growth chamber. Of the 208 accessions selected in the initial screen, 10 did not produce viable seed, leaving 198 accessions retested. In the seedling stage, a total of 164 accessions displayed a, MR to R phenotype (Table 2). In all, 24 accessions tested MS and 20 accessions tested S even though these accessions initially had tested MR to HR. None of the accessions displayed an HR response over all seedling tests.

All seedlings, including the 44 accessions that tested S or MS when tested as S1 seedlings, were allowed to grow to maturity and were reinoculated at the jointing or flag-leaf stage. Of the $198 \mathrm{ac}-$ cessions that were tested for resistance in the adult plant stage, 181 showed moderate to high resistance (Table 3 ). The majority of the tested accessions $(51 \%)$ had the same resistance reaction in both replications. For 45 accessions, the adult plant resistance phenotype was based on only one observation because one of the replications died before testing. Minor differences (one level of

Table 1. Number of accessions per wild oat species screened for crown rust resistance

\begin{tabular}{llc}
\hline Ploidy level, species & Genome & $\begin{array}{c}\text { Number of } \\
\text { accessions }\end{array}$ \\
\hline Diploid & AsAs & 2 \\
Avena atlantica & AdAd & 4 \\
A. damascena & CpCp & 6 \\
A. eriantha & AsAs & 1 \\
A. hirtula & A1A1 & 12 \\
A. longiglumis & $\ldots$ & 2 \\
A. ventricosa & AsAs & 5 \\
A. wiestii & & \\
Tetraploid & AABB & 12 \\
A. agadiriana & AABB & 49 \\
A. barbata & AACC & 38 \\
A. magna & AACC & \\
A. murphyi & & 2 \\
Hexaploid & AACCDD & 29 \\
A. sterilis & & 332 \\
Undetermined & $\ldots$ & \\
Avena spp. & $\ldots$ & \\
Total & &
\end{tabular}

a Genomic labeling according to Thomas (22). 
resistance) in resistance phenotype between both replications were observed in 53 accessions.

\section{Discussion}

This study revealed high levels of broad-spectrum resistance (resistance to a highly diverse population of $P$. coronata) to crown rust in wild oat accessions from Morocco. In all, 150 (47\%) of the accessions were resistant in both seedling and adult plant stage tests. Resistance was found in diploid species (47\% adult plus seedling) as well as in tetraploid species (48\% adult plus seedling). The high proportion of resistant accessions found in this study was surprising. In earlier studies on A. barbata accessions, where bulk inoculum from the St. Paul buckthorn nursery was also used, only 13 and $11.4 \%$ percent of the tested accessions presented moderate resistance or higher $(3,4)$. These accessions were collected in the Mediterranean region, including Morocco, where $8 \%$ of the accessions were resistant in the adult plant stage. This is considerably lower than the 46 to $54 \%$ resistance found in the group of A. barbata accessions tested in this study. The high proportion of resistant accessions in this study likely cannot be explained by a lack of virulent races in the bulk inoculum used. The inoculum from the St. Paul buckthorn nursery is very diverse (3) and comparable with that used in the earlier screenings of A. barbata. The only explanation we can offer at this moment is that the accessions from the earlier studies were collected at different times and at different geographical locations than the ones used in this study.

Although 11 wild oat species are represented in this study, the largest segment of the accessions is made up by A. barbata $(n=$ $179)$, followed by A. magna $(n=40)$ and A. murphyi $(n=38)$. These groups also contained the highest number of resistant accessions in this study. The tetraploid A. barbata had the largest number of accessions with resistance (MR or R) in both the seedling and adult plant stage, with 97 accessions (54\% of total A. barbata accessions). Of the A. murphyi group, 32 accessions (84\%) and, of the $A$. magna group, 20 accessions (50\%) showed MR to $\mathrm{R}$ reactions in both the seedling and adult plant stage.

Adult plant resistance is sometimes defined as resistance that is expressed at the adult plant stage but is not expressed at the seedling stage (2). In this study, we did not make this distinction of adult plant resistance that is only expressed at the adult plant stage.

Table 2. Number of accessions in a resistance phenotype class in the seedling tests, classified by wild oat species

\begin{tabular}{|c|c|c|c|c|c|c|c|}
\hline \multirow[b]{2}{*}{ Wild oat species } & \multicolumn{5}{|c|}{ Seedling evaluation ${ }^{a}$} & \multirow[b]{2}{*}{ Total resistant ${ }^{\mathrm{b}}$} & \multirow[b]{2}{*}{ Total screened $^{\mathrm{c}}$} \\
\hline & $\mathbf{S}$ & MS & MR & $\mathbf{R}$ & HR & & \\
\hline Avena agadiriana & 8 & 2 & 2 & 0 & 0 & 2 & 12 \\
\hline A. atlantica & 0 & 0 & 1 & 1 & 0 & 2 & 2 \\
\hline A. barbata & 66 & 30 & 37 & 46 & 0 & 83 & 179 \\
\hline A. damascena & 1 & 0 & 2 & 1 & 0 & 3 & 4 \\
\hline A. eriantha & 6 & 0 & 0 & 0 & 0 & 0 & 6 \\
\hline A. hirtula & 0 & 0 & 0 & 1 & 0 & 1 & 1 \\
\hline A. longiglumis & 0 & 3 & 3 & 6 & 0 & 9 & 12 \\
\hline A. magna & 14 & 7 & 8 & 11 & 0 & 19 & 40 \\
\hline A. murphyi & 4 & 5 & 21 & 8 & 0 & 29 & 38 \\
\hline Avena spp. & 11 & 4 & 10 & 4 & 0 & 14 & 29 \\
\hline A. sterilis & 1 & 1 & 0 & 0 & 0 & 0 & 2 \\
\hline A. ventricosa & 1 & 1 & 0 & 0 & 0 & 0 & 2 \\
\hline A. wiestii & 2 & 1 & 2 & 0 & 0 & 2 & 5 \\
\hline Total & 114 & 54 & 86 & 78 & 0 & 164 & 332 \\
\hline
\end{tabular}

${ }^{a}$ Results of the initial screening and the subsequent seedling tests combined. Resistance phenotype is based on the initial screening of seedlings and the subsequent testing of two offspring seedlings. The most susceptible phenotype is used in cases where resistance phenotype varied over the different tests. S = susceptible, large to moderately large pustules with little or no chlorosis; MS = moderately susceptible, moderately large pustules surrounded by extensive chlorosis; MR = moderately resistant, small pustule surrounded by chlorosis; $\mathrm{R}=$ resistant, chlorotic or necrotic flecking; and HR = highly resistant, no visible reaction.

b Total resistant accessions (MR, R, and HR).

${ }^{\mathrm{c}}$ Total accessions screened.

Table 3. Number of accessions in a resistance phenotype class in the adult plant evaluations, classified by wild oat species

\begin{tabular}{|c|c|c|c|c|c|c|c|c|}
\hline \multirow[b]{2}{*}{ Wild oat species } & \multicolumn{5}{|c|}{ Adult plant evaluation ${ }^{a}$} & \multirow[b]{2}{*}{ Adult resistant ${ }^{\mathrm{b}}$} & \multirow[b]{2}{*}{ Screened for adult ${ }^{\mathbf{c}}$} & \multirow[b]{2}{*}{ Total screened $^{\mathrm{d}}$} \\
\hline & $\mathbf{S}$ & MS & MR & $\mathbf{R}$ & HR & & & \\
\hline Avena agadiriana & 0 & 0 & 2 & 0 & 0 & 2 & 2 & 12 \\
\hline A. atlantica & 0 & 0 & 1 & 1 & 0 & 2 & 2 & 2 \\
\hline A. barbata & 4 & 1 & 30 & 57 & 10 & 97 & 102 & 179 \\
\hline A. damascena & 0 & 0 & 0 & 1 & 2 & 3 & 3 & 4 \\
\hline A. eriantha & 5 & 1 & 0 & 0 & 0 & 0 & 6 & 6 \\
\hline A. hirtula & 0 & 0 & 0 & 1 & 0 & 1 & 1 & 1 \\
\hline A. longiglumis & 0 & 0 & 2 & 3 & 4 & 9 & 9 & 12 \\
\hline A. magna & 0 & 0 & 7 & 12 & 1 & 20 & 20 & 40 \\
\hline A. murphyi & 2 & 0 & 15 & 16 & 1 & 32 & 34 & 38 \\
\hline Avena sp. & 1 & 1 & 3 & 7 & 2 & 12 & 14 & 29 \\
\hline A. sterilis & 0 & 0 & 1 & 0 & 0 & 1 & 1 & 2 \\
\hline A. ventricosa & 0 & 0 & 0 & 0 & 0 & 0 & 0 & 2 \\
\hline A. wiestii & 1 & 1 & 1 & 1 & 0 & 2 & 4 & 5 \\
\hline Total & 13 & 4 & 62 & 99 & 20 & 181 & 198 & 332 \\
\hline
\end{tabular}

${ }^{a} \mathrm{~S}=$ susceptible, large to moderately large pustules with little or no chlorosis; MS = moderately susceptible, moderately large pustules surrounded by extensive chlorosis; $\mathrm{MR}=$ moderately resistant, small pustule surrounded by chlorosis; $\mathrm{R}=$ resistant, chlorotic or necrotic flecking; and HR = highly resistant, no visible reaction. Resistance in the adult plant stage is based on two offspring per accession. The most susceptible phenotype is used in cases where resistance phenotype varied between the two offspring plants.

b Total adult resistant accessions.

${ }^{\mathrm{c}}$ Total screened for adult resistance.

d Total accessions screened. 
Resistance to crown rust at the adult plant stage is more desirable than resistance at the seedling stage for oat production. For practical purposes, however, we screened at the seedling stage first, ending up with accessions with resistance expressed at the seedling as well as adult plant stage. However, we did find some accessions with different resistance responses between seedling and adult plant stage. A few accessions (A. barbata PI657286, Avena sp. PI657481, and A. wiestii PI657512) presented an MR to R response in the seedling and an $\mathrm{S}$ response in the adult plant stage, indicative of $R$ genes that are functional at a specific development stage. A few accessions were only MR or MS in the seedling stage and HR in the adult plant stage (A. barbata PI657283 and PI657350, A. damascena PI657472, A. longiglumis PI657389, and Avena sp. PI657425). This might indicate that there are two or more $R$ genes present in that given accession, with one becoming functional at the adult plant stage, or there may be a single $R$ gene that varies in effectiveness in a stage-specific manner. More accessions displayed an HR reaction in the adult plant stage than in the seedling stage. In the seedling stage, none of the accessions displayed a consistent HR reaction over the different seedling tests but, in the adult plant stage, 20 accessions showed an HR reaction across all the tests.

The new resistance sources from wild oat species can significantly contribute to future resistance breeding in oat. At least some of the $P c$ genes expressed in this study are likely identical, because all accessions were collected in the same geographical area. However, differences in resistance reaction phenotype were present, and resistance was found in different species. This result indicates at least some diversity of new broad-spectrum $R$ genes useful for introgressing into domestic oat. The NSGC database currently holds 11,294 wild oat accessions, of which 1,490 are diploid or tetraploid Avena spp. To our knowledge, approximately half of the diploid and tetraploid wild oat species in the NSGC database has now been screened for crown rust resistance (402 A. barbata screened by Carson [3] and the 332 accessions of wild Avena spp. screened here). There are more accessions of wild Avena spp. in other plant germplasm collections in the world (13). It is not clear how many of the accessions are duplicates but it is reasonable to think that these collections contain new sources of crown rust resistance as well. A screening of 1,099 A. barbata accessions of the Canadian Plant Gene Resources Center resulted in 125 accessions exhibiting broad-spectrum resistance. More accessions of wild Avena spp. should be collected to fully represent the diversity of Avena spp. in germplasm collections. Species such as A. murphyii, A. magna, and A. insularis are presently underrepresented in world collections and are in need of preservation because their native habitats are threatened (13).

The challenge is now to introgress the newly found $R$ genes into cultivated oat. Traditional hybridization methods are the preferred method in oat, given that a transgenic approach will not be readily accepted in this crop with a natural or "organic" image. A. sativa (genome AACCDD) and its nonhexaploid relatives have limited compatibility with each other but it is possible to cross most oat species with different ploidy levels. Leggett and Thomas (10) assigned the species of Avena into three gene pools, based on the ease of introgression of the genes from the wild species into the cultivated oat. All hexaploid species form the primary gene pool in this system. Tetraploid species with the AACC genome form the secondary gene pool, based on the ability of the F1 of crosses with hexaploids to set seed and contain recombinant chromosomes. The resistance found in this study in wild oat species with the AACC genome, 52 resistant accessions of A. magna and A. murphyi, should be relatively easy to transfer into A. sativa. All other tetraploids and the diploids form the tertiary gene pool and introgression of traits from these wild species is more challenging. Crown rust $R$ genes from the diploid black oat, A. strigosa (genome $\mathrm{A}_{\mathrm{S}} \mathrm{A}_{\mathrm{S}}$ ), have nevertheless been successfully introgressed into $A$. sativa (20). The majority of resistant accessions found in this study are of wild species A. barbata, with 97 resistant accessions. The tetraploid $A$. barbata (AABB) crosses readily with $A$. sativa but the resulting pentaploid is sterile. Partial fertility can be restored if the $\mathrm{F} 1$ is treated with colchicine to double the chromosomes, producing a decaploid that can then be backcrossed to A. sativa (unpublished data).

Successful introgression of resistance into A. sativa is further complicated by the lack of recombination between the chromosomes of the hexaploid A. sativa and alien chromosomes from the wild species. The functioning of so-called pairing genes restricts the amount of pairing between chromosomes of A. sativa and wild oat (24). Spontaneous translocations do occur and depend on the amount of synteny between the alien chromosome and its $A$. sativa homologue in the region of the resistance locus. Two strategies can be used to induce recombination. Irradiation can be used to introduce random transfers of chromosome segments. However, the side effect is often cytological instability and low fertility (1). A more elegant technique involves the diploid A. longiglumis genotype CW57. CW57 apparently contains a factor similar to the Phl locus in wheat, which is capable of suppressing the regular activity of the pairing genes during meiosis in A. sativa $(19,23)$. The crown rust $R$ genes $P c 91$ from A. magna and Pc94 from A. strigosa have been stably incorporated into cultivars using this approach $(1,7,15,16)$. This approach will most likely also work for incorporating crown rust resistance from other tetraploid oat accessions.

Suppression of resistance in interploidy crosses has been known to occur occasionally when transferring crown rust resistance from alien species into A. sativa. Pc94 resistance from A. strigosa and $P c 62$ resistance from $A$. sterilis is suppressed by the presence of $P c 38$ or some factor closely linked to it $(6,25)$. The very high level of resistance of $A$. murphyi $\mathrm{P} 12$ was also reported to be completely suppressed when crossed to A. sativa and was not recovered in subsequent backcross generations when attempting to transfer it to cultivated oat (20). Suppression of $R$ gene expression seems to occur in many polyploid species and becomes apparent in interploidy crosses. However, suppression of resistance seems to be a matter of specific combinations of genotypes and can be circumvented when using a different (susceptible) parent for the interploidy cross.

There is a need for new $R$ genes because all of the known $P c R$ genes have been overcome by the pathogen. This study has added many potential new $P c$ genes for use in oat cultivars by screening wild oat accessions. Particularly of interest are the new resistance sources found in A. magna and A. murphyi, because these are expected to be relatively easy to introgress into cultivated oat. These accessions with the newly found $R$ genes are available for introgressing into cultivated oat and development of varieties. Ideally, there will be multiple $P c$ gene combinations in cultivars so that oat crown rust resistance does not depend exclusively on $P c$ genes deployed singly.

\section{Acknowledgments}

We thank J. Ochocki for technical assistance and P. Olivera and L. Szabo for providing feedback on the manuscript.

\section{Literature Cited}

1. Aung, T., Chong, J., and Leggett, M. 1996. The transfer of crown rust resistance gene Pc94 from a wild diploid to cultivated hexaploid oat. Pages 167-171 in: Proc. 9th Eur. Mediterr. Cereal Rusts Powdery Mildews Conf. G. H. J. Kema, R. E. Niks, and R. A. Daamen, eds. European and Mediterranean Cereal Rust Foundation, Wageningen and Lunteren, The Netherlands.

2. Cabral, A. L., Singh, D., and Park, R. F. 2011. Identification and genetic characterisation of adult plant resistance to crown rust in diploid and tetraploid accessions of Avena. Ann. Appl. Biol. 159:220-228.

3. Carson, M. L. 2009. Broad-spectrum resistance to crown rust, Puccinia coronata $\mathrm{f}$. sp. avenae, in accessions of the tetraploid slender oat, Avena barbata. Plant Dis. 93:363-366.

4. Carson, M. L. 2010. Additional sources of broad-spectrum resistance to Puccinia coronata f. sp. avenae from Canadian accessions of Avena barbata. Plant Dis. 94:1405-1410.

5. Carson, M. L. 2011. Virulence in oat crown rust (Puccinia coronata f. sp. avenae) in the United States from 2006 through 2009. Plant Dis. 95:15281534

6. Chong, J., and Aung, T. 1996. Interaction of the crown rust resistance gene $P c 94$ with several $P c$ genes. Pages $172-175$ in: Proc. 9 th Eur. Mediterr. Ce- 
real Rusts Powdery Mildews Conf. G. H. J. Kema, R. E. Niks, and R. A. Daamen, eds. European and Mediterranean Cereal Rust Foundation, Wageningen and Lunteren, The Netherlands.

7. Fetch, J. W. M., Duguid, S. D., Brown, P. D., Chong, J., Fetch, T. G., Haber, S. M., Menzies, J. G., Ames, N., Noll, J., Aung, T., and Stadnyk, K. D. 2007. Leggett oat. Can. J. Plant Sci. 87:509-512.

8. Kulcheski, F. R., Graichen, F. A. S., Martinelli, J. A., Locatelli, A. B., Federizzi, L. C., and Delatorre, C. A. 2010. Molecular mapping of Pc68, a crown rust resistance gene in Avena sativa. Euphytica 175:423-432.

9. Leggett, J. M. 1992. Classification and speciation in Avena. Pages 29-52 in: Oat Science and Technology. H. G. Marshall and M. E. Sorrells, eds. American Society of Agronomy and Crop Science Society of America, Madison, WI.

10. Leggett, J. M., and Thomas, H. 1995. Oat evolution and cytogenetics. Pages 120-149 in: The Oat Crop: Production and Utilization. R. W. Welch, ed. Chapman and Hall, London.

11. Loskutov, I. G. 2004. Using of wild species genetic diversity in plant breeding. In: 4th Int. Crop Sci. Congr. Brisbane, Australia.

12. Loskutov, I. G. 2008. On evolutionary pathways of Avena species. Genet. Resour. Crop Evol. 55:211-220.

13. Loskutov, I. G., and Rines, H. W. 2011. Avena. Pages 109-183 in: Wild Crop Relatives: Genomic and Breeding Resources: Cereals. C. Kole, ed. Springer, Heidelberg.

14. Martens, J. W., and Dyck, P. L. 1989. Genetics of resistance to rust in cereals from a Canadian perspective. Can. J. Plant Pathol. 11:78-85.

15. McCartney, C. A., Stonehouse, R. G., Rossnagel, B. G., Eckstein, P. E., Scoles, G. J., Zatorski, T., Beattie, A. D., and Chong, J. 2011. Mapping of the oat crown rust resistance gene Pc91. Theor. Appl. Genet. 122:317-325.
16. McMullen, M. S., Doehlert, D. C., and Miller, J. D. 2005. Registration of 'Hifi' oat. Crop Sci. 45:1664-1664.

17. Moore, M. B. 1966. Buckthorns and oat breeding for resistance to crown rust. (Abstr.). Phytopathology 56:891.

18. Politowski, K., and Browning, J. A. 1975. Effect of temperature, light, and dew duration on relative numbers of infection structures of Puccinia coronata avenae. Phytopathology 65:1400-1404.

19. Rajhathy, T., and Thomas, H. 1972. Genetic control of chromosome pairing in hexaploid oats. Nat. New Biol. 239:217-219.

20. Rines, H. W., Porter, H. L., Carson, M. L., and Ochocki, G. E. 2007. Introgression of crown rust resistance from diploid oat Avena strigosa into hexaploid cultivated oat $A$. sativa by two methods: direct crosses and through an initial 2x·4x synthetic hexaploid. Euphytica 158:67-79.

21. Simons, M. D. 1985. Crown rust. Pages 131-172 in: The Cereal Rusts: Diseases, Distribution, Epidemiology, and Control. A. P. Roelfs and R. Bushnell, eds. Academic Press, Orlando, FL.

22. Thomas, H. 1992. Cytogenetics of Avena. In: Oat Science and Technology. H. G. Marshall and M. E. Sorrells, eds. American Society of Agronomy and Crop Science Society of America, Madison, WI.

23. Thomas, H., and Alansari, N. 1988. Genotypic control of chromosomepairing in amphiploids involving the cultivated oat Avena sativa L. Euphytica 37:37-45.

24. Thomas, H., Powell, W., and Aung, T. 1980. Interfering with regular meiotic behavior in Avena sativa as a method of incorporating the gene for mildew resistance from Avena barbata. Euphytica 29:635-640.

25. Wilson, W. A., and McMullen, M. S. 1997. Dosage dependent genetic suppression of oat crown rust resistance gene Pc-62. Crop Sci. 37:16991705. 